\title{
EFEITO DA APLICAÇÃO DE POTÁSSIO E SÓDIO NAS CARACTERÍSTICAS DO LENHO DE ÁRVORES DE Eucalyptus grandis W.HILL, AOS 24 MESES DE IDADE
}

\author{
Carlos Roberto Sette Júnior ${ }^{1}$, Mário Tomazello Filho ${ }^{2}$, Carlos Tadeu dos Santos Dias ${ }^{3}$, \\ Matheus Peres Chagas ${ }^{4}$, Jean Paul Laclau ${ }^{5}$ \\ ${ }^{1}$ Eng. Florestal, M.Sc., Doutorando em Recursos Florestais, ESALQ/USP, Piracicaba, SP, Brasil - crjunior@esalq.usp.br \\ ${ }^{2}$ Eng. Agrônomo, Dr., Depto. de Ciências Florestais, ESALQ/USP, Piracicaba, SP, Brasil - mtomazel@esalq.usp.br \\ ${ }^{3}$ Eng. Agrônomo, Dr., Depto. de Ciências Exatas, ESALQ/USP, Piracicaba, SP, Brasil - ctsdias@ esalq.usp.br \\ ${ }^{4}$ Eng. Florestal, Mestrando em Recursos Florestais, ESALQ/USP, Piracicaba, SP, Brasil - mpchagas@esalq.usp.br \\ ${ }^{5}$ Eng. Florestal, Dr., CIRAD/USP, Piracicaba, SP, Brasil - jplaclau @ esalq.usp.br
}

Recebido para publicação: 21/11/2007 - Aceito para publicação: 16/10/2008

\begin{abstract}
Resumo
O presente trabalho teve como objetivo avaliar as características anatômicas e a densidade aparente e básica do lenho de árvores de Eucalyptus grandis com 24 meses de idade, plantadas no espaçamento de $3 \times 2 \mathrm{~m}$, sem fertilização (testemunha) e fertilizadas com potássio (T2) e sódio (T3). De cada tratamento foram cortadas 8 árvores de eucalipto, de acordo com a distribuição de área basal, e coletadas amostras do lenho em diferentes porcentagens da sua altura total. $\mathrm{Na}$ avaliação das dimensões dos vasos e fibras e da densidade aparente por densitometria de raios X, foram utilizadas as amostras do lenho do DAP, e para a densidade básica todas as amostras do lenho. Os resultados mostraram que as densidades aparente e básica média do lenho das árvores de eucalipto não foram significativas nos 3 tratamentos, sendo os perfis radiais de densidade aparente característicos de madeira juvenil de árvores de eucalipto. A aplicação dos fertilizantes minerais (potássio e sódio) induziu alterações nas dimensões das fibras, responsáveis pela sustentação do tronco das árvores de eucalipto, não sendo detectadas diferenças significativas para os elementos de vaso.

Palavras-chave: Anatomia da madeira; densidade; eucalipto; fertilização.
\end{abstract}

\begin{abstract}
Effect of pottassium and sodium application on the wood characteristics of Eucalyptus grandis W. Hill, at 24 months old-trees. The present work had as objective to evaluate the wood anatomical characteristics and the wood apparent and basic density of Eucalyptus grandis 24 months old-trees, planted in the spacing of $3 \times 2 \mathrm{~m}$, without fertilization (control) and fertilized with potassium (T2) and sodium (T3). For each treatment they cut 8 eucalyptus trees, acoording to basal area distribution, and collected wood samples at different percentages of tree heights. In the evaluation of the vessel and fiber dimensions and the wood apparent density by X-ray densitometry they were used wood samples at $\mathrm{DBH}$ and for wood basic density they were used all the wood samples. The results showed that the apparent and basic wood density values were not significant in the 3 treatments, and the radial wood density profiles were characteristic of juvenile wood of eucalyptus trees. The application of mineral fertilizers (potassium and sodium) induced alterations in the fiber dimensions, responsible for the eucalypt tree sustentation and not significant differences for the vessels elements were detected. Keywords: Wood anatomy; wood density; eucalypt; fertilization.
\end{abstract}

\section{INTRODUÇÃO}

Atualmente, a possibilidade de escassez de madeira na próxima década e a redução da pressão sobre as florestas naturais têm proporcionado uma inversão na tendência decrescente nas taxas de reflorestamento anuais, mostrando forte reação no ano de 2005, com 500.000 ha reflorestados em várias regiões do país. Para atender a demanda projetada, as universidades, instituições de pesquisa e as empresas florestais têm desenvolvido pesquisas para o aumento da produtividade das plantações 
florestais, com investimentos no melhoramento genético e clonagem, preparo do solo e fertilização mineral das árvores (TOMAZELLO FILHO, 2006).

O efeito significativo da fertilização mineral em árvores de eucalipto é reconhecido há algumas décadas, desde as pesquisas iniciais, realizadas por Mello (1968) com árvores de Eucalyptus saligna, aos 2 anos, em solos pobres e ácidos do cerrado, obtendo uma diferença de $33 \mathrm{~m} / \mathrm{ha}$ na produção de madeira nas árvores adubadas e não adubadas. Posteriormente, outros autores verificaram aumentos de 67 a $118 \%$ do volume de madeira de árvores de Eucalyptus saligna e E. grandis adubadas com potássio no cerrado do estado de Minas Gerais (BARROS et al., 1981 citado por SILVEIRA; MALAVOLTA, 2003) e no sul do estado de São Paulo (GAVA, 1997), respectivamente.

Enquanto a aplicação de fertilização mineral aumenta significativamente a taxa de crescimento das árvores de eucalipto, são escassas as informações sobre o seu efeito nas propriedades da madeira. Os resultados de pesquisas referentes ao efeito de fertilizantes minerais nas propriedades da madeira de árvores de espécies de eucalipto de idades avançadas (até 30 anos) indicam o aumento (VIGNERON et al., 1995; BOUVET et al., 1999) e a redução (SHIMOYAMA et al., 1989; WASHUSEN et al., 2005) da densidade do lenho. Em árvores jovens (1-3 anos) de eucalipto destacam-se os resultados de Bamber et al. (1982) e Pereira et al. (1990), que não verificaram efeito significativo da fertilização mineral na densidade do lenho e na dimensões das fibras. Nesse aspecto, Raymond (1998) e Beadle et al. (2001) destacam a importância da análise das alterações das propriedades da madeira das árvores de eucalipto com taxas de crescimento elevadas pela fertilização mineral.

Diante do exposto, o presente trabalho teve como objetivo avaliar o efeito da aplicação de potássio e sódio nas características anatômicas e físicas do lenho de árvores de Eucalyptus grandis aos 24 meses.

\section{MATERIAL E MÉTODOS}

\section{Local}

O experimento foi instalado na Estação Experimental de Ciências Florestais de Itatinga/SP, do Departamento de Ciências Florestais da ESALQ/USP, localizada a $23^{\circ} 10^{\prime} \mathrm{S}$ e $48^{\circ} 40^{\prime} \mathrm{W}$, com $857 \mathrm{~m}$ de altitude. O clima da região, segundo Köppen, é caracterizado como mesotérmico úmido (Cwa), com precipitação média anual de $1.635 \mathrm{~mm}$. A temperatura média do mês mais frio é $12,8{ }^{\circ} \mathrm{C}$ e a média do mês mais quente é de $19,4^{\circ} \mathrm{C}$. O solo é do tipo Latossolo Vermelho Amarelo distrófico de textura média (200 g. $\mathrm{kg}^{-1}$ de argila), cuja litologia é composta por arenito, formação Marília, pertencendo ao Grupo Bauru.

\section{Delineamento experimental}

As características do lenho foram avaliadas em árvores de eucalipto coletadas do projeto "Resposta do Eucalyptus grandis à adubação potássica e à substituição do potássio por sódio", implantado em abril/2004, em delineamento experimental em blocos casualizados com 3 tratamentos $\left(\mathbf{T}_{\mathbf{1}}\right.$ : controle; $\mathbf{T}_{2}$ : aplicação de $140 \mathrm{~kg} / \mathrm{ha}$ de $\mathrm{K}$ na forma de $\mathrm{K}_{2} \mathrm{O}$ no plantio, 6 e 12 meses; e $\mathbf{T}_{3}$ : aplicação de $174,4 \mathrm{~kg} / \mathrm{ha}$ de $\mathrm{Na}$ na forma de $\mathrm{NaCl}$ no plantio, 6 e 12 meses) e 4 blocos. Todos os tratamentos receberam $80 \mathrm{~kg} \cdot \mathrm{ha}^{-1}$ de $\mathrm{P}_{2} \mathrm{O}_{5}, 45 \mathrm{~kg} \cdot \mathrm{ha}^{-1}$ de $\mathrm{N}\left(\mathrm{NH}_{4}(\mathrm{SO} 4)_{2}\right)$ e $45 \mathrm{~kg} \cdot \mathrm{ha}^{-1}$ de FTE (BR-12) (LACLAU et al., 2007).

\section{Seleção das árvores e coleta das amostras do lenho}

Foram selecionadas 8 árvores de eucalipto por tratamento (total de 24 árvores), de acordo com a distribuição de área basal, que foram abatidas, desramadas, para o corte de discos de madeira em 6 diferentes posições longitudinais do tronco (base, DAP, 3, 6, 9 e $12 \mathrm{~m}$ da altura total).

\section{Variação da densidade aparente do lenho por densitometria de raios $\mathbf{X}$}

Dos discos de lenho do DAP foram cortadas amostras diametrais $(20 \times 10 \mathrm{~mm}$, largura $\mathrm{x}$ altura), coladas em suporte de madeira e seccionadas no sentido transversal $(2,0 \mathrm{~mm}$ de espessura) em aparelho de dupla serra circular paralela (Figura 1-A). As seções transversais do lenho (Figura 1-B) foram acondicionadas em câmara de climatização $\left(12 \mathrm{~h}, 20^{\circ} \mathrm{C}, 50 \%\right.$ UR), até atingir $12 \%$ de umidade. As amostras transversais do lenho e a cunha de calibração de acetato de celulose foram dispostas sobre 
suporte com filme de raios X (Kodak, Diagnostic Film T-Mat, 240x180 mm), transferidas para equipamento de raios X (Hewlett Packard, Faxitron $43805 \mathrm{~N} ; 1,20 \mathrm{~m}$ distância da fonte de raios X-filme) e radiografadas ( $5 \mathrm{~min}, 16 \mathrm{Kv}, 3 \mathrm{~mA}$ ) (Figura 1-C, D). Os filmes radiográficos das amostras de madeira + cunha de calibração foram revelados em aparelho Macrotec MX-2 (MOTHE, 1998) e digitalizados em scanner Hewlett Packard ScanJet 6100C/T, na resolução de 1000 dpi, em escala de cinza de 256 graus. $\mathrm{Na}$ imagem digitalizada foram feitas comparações da escala de cinza das amostras de madeira com a da cunha de calibração, sendo determinados os valores de densidade, convertidos para arquivo tipo DEN, através do software CRAD. Na sequência, o arquivo DEN foi lido com auxílio do software CERD, considerando 500 x 10 (comprimento x largura) determinações de densidade para os segmentos de $25 \%$ do comprimento das amostras do lenho (Figura 1-E). As análises diametrais das amostras originam um arquivo tipo TXT contendo os valores pontuais de densidade e os respectivos gráficos dos perfis de densidade aparente do lenho das árvores de eucalipto (Figura 1-F). A partir dos perfis radiais de densidade aparente do lenho, foram determinadas as densidades aparente média, máxima e mínima do lenho para cada tratamento (TOMAZELLO FILHO, 2006).

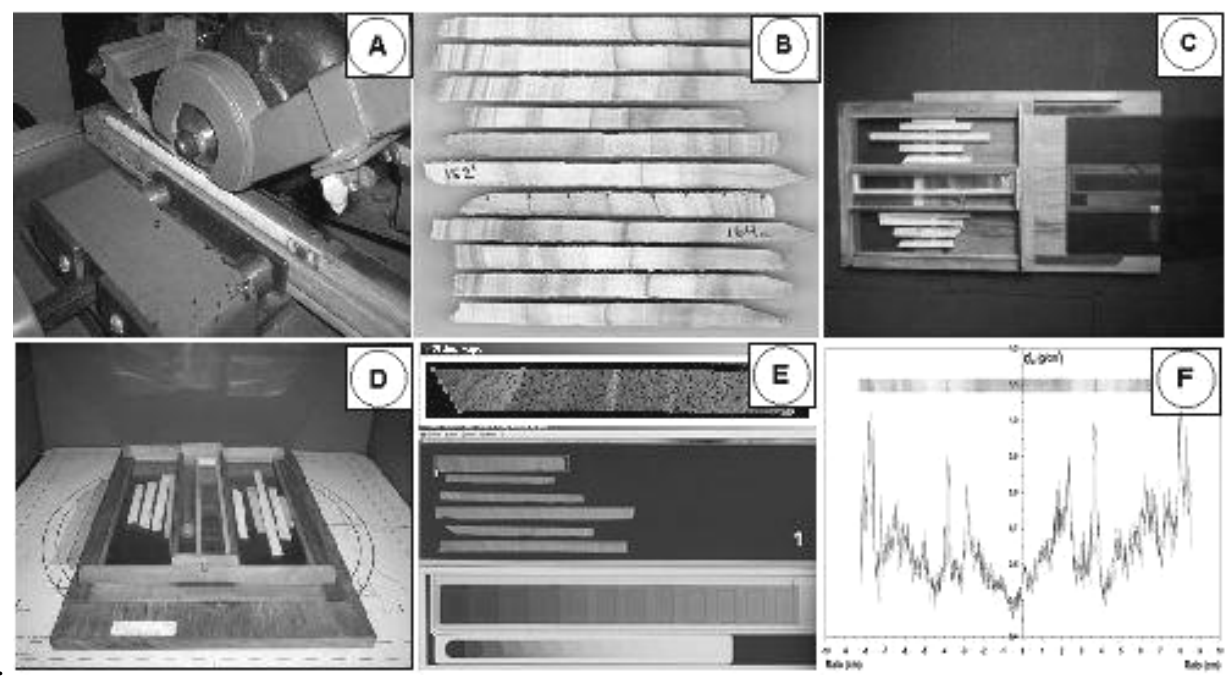

Figura 1. A metodologia de raios $\mathrm{X}$ e a obtenção das imagens radiográficas do lenho das árvores de eucalipto: (A) corte das amostras em equipamento de dupla serra; (B) seções transversais das amostras; (C) amostras de madeira e cunha de calibração de acetato de celulose sobre o filme radiográfico; (D) irradiação das amostras em equipamento de raios X; (E) imagem radiográfica e análise nos programas CRAD e CERD; (F) perfil da densidade aparente e respectiva amostra do lenho. Fonte: Tomazello Filho (2006).

Figure 1. The X-ray methodology and eucalypt wood radiographs: (A) wood samples are cut with double parallel circular saws; (B) wood cross-sections; (C) wood samples and cellulose acetate wedge on radiographic film; (D) irradiation of the wood samples in X-ray equipment; (E) wood radiographic image and analysis in CRAD and CERD programs; (F) wood density profile and respective wood cross-section. From: Tomazello Filho (2006).

\section{Variação da densidade básica do lenho no sentido longitudinal do tronco}

Discos do lenho da base, DAP, 3, 6, 9 e $12 \mathrm{~m}$ do tronco das árvores foram selecionados e imersos em água até a sua saturação completa, para obtenção dos pesos úmidos $\left(\mathrm{P}_{\mathrm{u}}\right)$, imersos $\left(\mathrm{P}_{\mathrm{i}}\right)$ e secos $\left(\mathrm{P}_{\mathrm{s}}\right)$, determinando-se a densidade básica $\left(\mathrm{d}_{\mathrm{B}}\right)$ pelo método da balança hidrostática (Equação 1). Os valores de densidade básica do lenho foram utilizados na determinação da sua variação longitudinal, da densidade básica média ponderada e da sua relação com os níveis de fertilização.

$$
d_{B}=\frac{P_{s}}{P_{u}-P_{i}}
$$




\section{Variação da estrutura anatômica do lenho: dimensões dos vasos e fibras}

Dos discos do lenho do DAP foram cortadas amostras transversais e, destas, corpos de prova radiais $(10 \times 10 \mathrm{~mm}$, largura $\mathrm{x}$ altura). Os corpos de prova do lenho foram saturados em água, amolecidos em água à ebulição, fixados em micrótomo de deslize, para serem cortadas seções transversais (15-20 ųm de espessura). Estas foram clarificadas (água cândida, 1:1), lavadas (água destilada, ácido acético 1\%), desidratadas (série alcoólica, 30-100\%), lavadas (xilol), coradas (safranina) e montadas com bálsamo de Canadá em lâminas de vidro. Das lâminas histológicas seriadas das seções transversais do lenho foram coletadas imagens digitais a cada $1 \mathrm{~cm}$ na direção medula-casca (3 imagens/posição/cm), com câmera digital acoplada a microscópio de luz (50x), para a determinação do diâmetro tangencial, da frequência e da área ocupada pelos vasos (equações 2 e 3), pela aplicação do programa de análise de imagens SAIM (Figura 2).

Frequência dos vasos $=\left(n^{0}\right.$ de vasos/área da imagem $) \times 100$

$\%$ de área de vasos = (área total vasos/área da imagem) $\times 100$
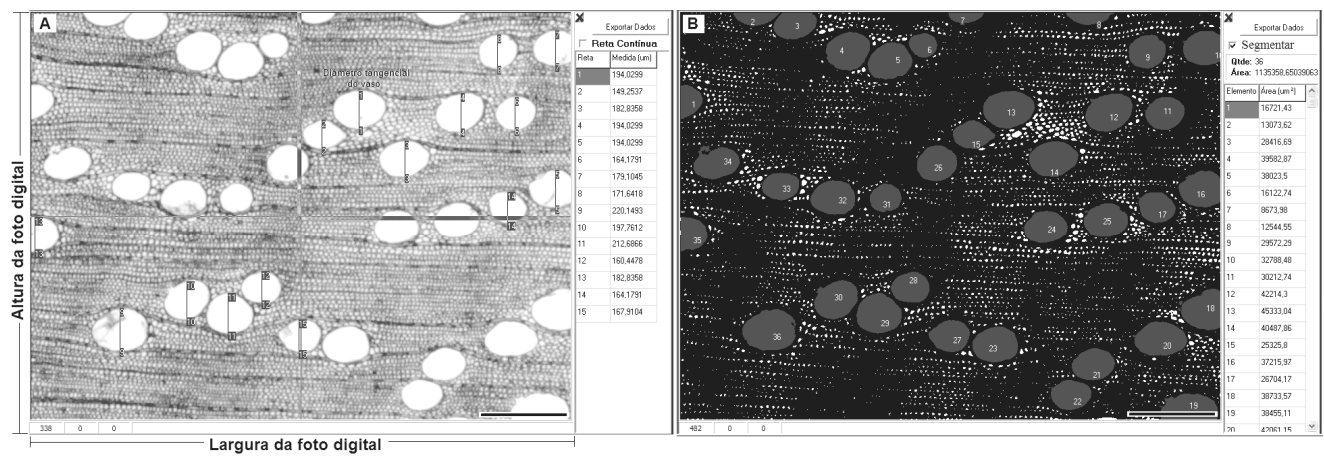

Figura 2. Seção transversal do lenho de Eucalyptus grandis para a avaliação do diâmetro e da frequência (A) e da área ocupada pelos vasos (B) em programa de análise de imagem SAIM. Barra $=300$ $\mu \mathrm{m}$.

Figure 2. Cross section of the Eucalyptus grandis wood for vessel diameter and frequency (A) and vessel area (B) evaluation in analysis image program SAIM. Bar $=300 \mu \mathrm{m}$.

Os corpos de prova radiais do lenho foram submetidos ao processo de maceração pelo método de Franklin, em tubos de ensaio (ácido acético + peróxido de hidrogênio -120 vol; 1:1) perfeitamente fechados e mantidos em estufa $\left(60^{\circ} \mathrm{C}, 48 \mathrm{~h}\right.$ ) (SASS, 1951). Da suspensão de células coradas (safranina + glicerina), foram montadas lâminas de vidro e coletadas as imagens das fibras em câmera digital acoplada em microscópio de luz. Foram mensurados o comprimento (ampliação de x25), a largura, a espessura da parede e o diâmetro do lume (ampliação de x400) das fibras (IAWA COMMITTEE, 1989), aplicando-se o programa de análise de imagens SAIM (Figura 3).
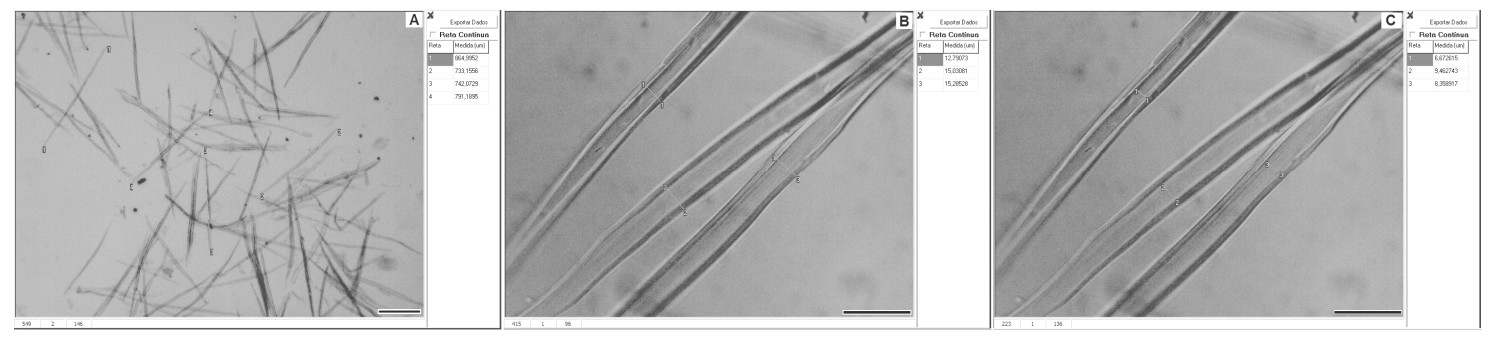

Figura 3. Lenho de Eucalyptus grandis dissociado para a avaliação do (A) comprimento, (B) largura e (C) diâmetro do lume da fibra em programa de análise de imagem SAIM. Barra $=300 \mu \mathrm{m}$.

Figure 3. Eucalyptus grandis wood dissociated to evaluation of the fiber (A) length, (B) diameter and (C) lumen diameter in image analysis program SAIM. Bar $=300 \mu \mathrm{m}$. 


\section{Análises estatísticas}

Nas análises estatísticas foi aplicado o programa SAS (SAS INSTITUTE INC, 1997), sendo aferidos os dados discrepantes, a heterogeneidade da variância e a escala pelo método de potência ótima de Box-Cox para a melhor transformação (BOX e COX, 1964). A análise de variância (ANOVA) foi aplicada para se verificar o efeito dos tratamentos e, quando significativo, o teste de Tukey a 95\% de probabilidade foi usado. A análise de regressão foi aplicada na variação radial e longitudinal das características da madeira, e no melhor modelo polinomial das equações de regressão foram considerados a significância (95\% de probabilidade), o coeficiente de determinação $\left(\mathrm{R}^{2}\right)$ e a distribuição dos resíduos.

\section{RESULTADOS E DISCUSSÃO}

\section{Variação radial da densidade aparente do lenho por densitometria de raios $\mathbf{X}$}

O modelo de variação radial da densidade aparente do lenho não mostrou diferenças significativas nas árvores de eucalipto dos três tratamentos (Figura 4). Os maiores valores de densidade aparente do lenho foram observados na medula $\left(0,80-1,20 \mathrm{~g} / \mathrm{cm}^{3}\right)$, devido à presença de substâncias de reserva (amido) nas células do parênquima axial, seguindo-se a sua redução e estabilização $(0,30$ $\left.0,50 \mathrm{~g} / \mathrm{cm}^{3}\right)$ até atingir a casca. $\mathrm{O}$ maior valor da densidade na medula e a oscilação do seu valor nos perfis densitométricos, em função dos elementos de vasos e das faixas de fibras de parede espessa têm sido verificados em lenho de eucalipto. Esses perfis densitométricos do lenho juvenil de árvores de eucalipto com 2 anos mostram diferenças com os obtidos para árvores de idade mais avançada (de 10-30 anos) por Oliveira (1997), Silva (2002), Silva et al. (2004), Alzate (2004), Benjamim (2006) e Tomazello Filho (2006), caracterizados pela nítida presença das madeiras juvenil, de transição e adulta.

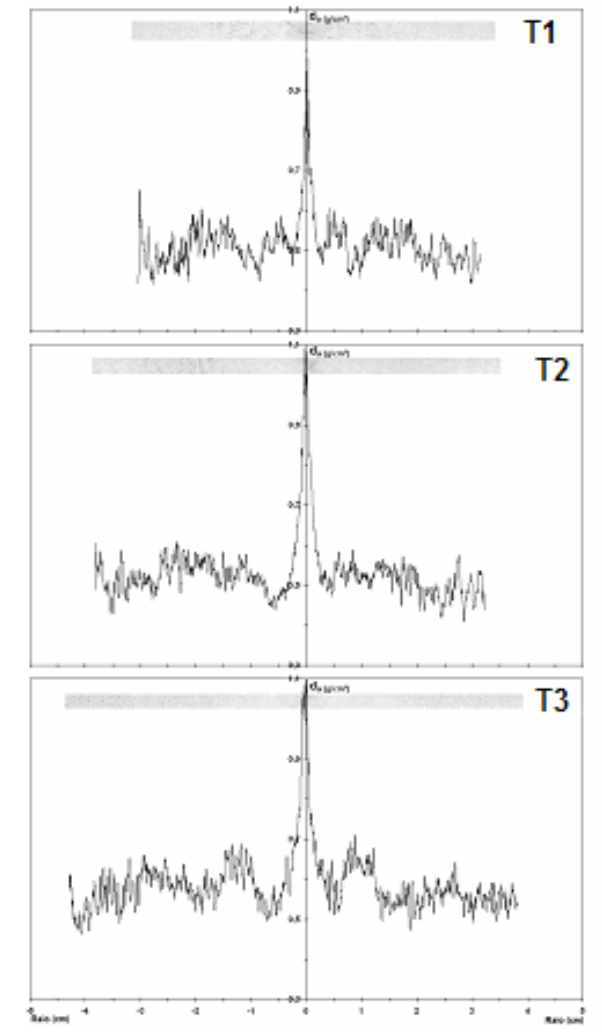

Figura 4. Perfil radial de densidade aparente do lenho das árvores de eucalipto (T1: testemunha; T2: potássio; T3: sódio).

Figure 4. Wood apparent density profile of the radial samples of the eucalypt trees (T1: control; T2: potassium; T3: sodium). 
A densidade aparente média do lenho das árvores de eucalipto dos tratamentos 1 (testemunha), 2 (aplicação de potássio) e 3 (aplicação de sódio) foi de $0,54,0,52$ e $0,53 \mathrm{~g} / \mathrm{cm}^{3}$, respectivamente. Os valores de densidade do lenho das árvores dos três tratamentos não foram significativos, corroborando os obtidos por Bamber, Horne e Graham-Higgs (1982) em árvores de E. grandis de 2,5 anos, de altas taxas de crescimento induzidas pelo preparo do solo e pela fertilização. No entanto, Shimoyama, Barrichelo (1989); Jacob e Balloni (1978); Andrade et al. (1994) e Washusen et al. (2005) indicaram uma redução da densidade do lenho das árvores de eucalipto fertilizadas.

A densidade aparente média do lenho foi cerca de $25 \%$ inferior às obtidas, pela mesma metodologia de densitometria de raios X, por Oliveira (1997), Benjamin (2006) e Tomazello Filho (2006), em função do efeito da idade das árvores e da formação da madeira adulta. A exemplo da densidade média, as densidades mínima e máxima aparente do lenho não foram significativas nos 3 tratamentos, indicando a sua homogeneidade, até a idade de 24 meses, nas árvores de eucalipto.

Tabela 1. Valores de densidade aparente média, máxima e mínima do lenho das árvores de eucalipto. Table 1. Mean, maximum and minimum wood density values of the eucalypt trees.

\begin{tabular}{lccc}
\hline \multirow{2}{*}{ Tratamento } & \multicolumn{3}{c}{ Densidade aparente $\left(\mathbf{g} / \mathbf{c m}^{\mathbf{3}}\right)$} \\
\cline { 2 - 4 } & Média & Máxima & Mínima \\
\hline 1 & 0,5409 a $(0,0278)$ & 0,9101 a $(0,1796)$ & 0,3757 a $(0,0420)$ \\
2 & 0,5243 a $(0,0437)$ & 0,9688 a $(0,2726)$ & 0,3716 a $(0,0529)$ \\
3 & 0,5328 a $(0,0525)$ & 0,9444 a $(0,1722)$ & 0,3772 a $(0,0870)$ \\
\hline
\end{tabular}

Médias seguidas de desvio padrão. Numa mesma linha, médias seguidas de pelo menos uma letra igual não diferem significativamente $(\mathrm{p}>0,05)$. Tratamento 1: testemunha; Tratamento 2: potássio; Tratamento 3: sódio.

\section{Variação da densidade básica do lenho no sentido longitudinal do lenho}

A densidade básica do lenho mostra um decréscimo até $3 \mathrm{~m}$ de altura do tronco nas árvores de eucalipto dos tratamentos $1\left(0,41 \rightarrow 0,39 \mathrm{~g} / \mathrm{cm}^{3}\right), 2\left(0,42 \rightarrow 0,38 \mathrm{~g} / \mathrm{cm}^{3}\right)$ e $3\left(0,40 \rightarrow 0,37 \mathrm{~g} / \mathrm{cm}^{3}\right)$, seguindose um aumento até os $9,0 \mathrm{~m}$ nos tratamentos $1\left(0,39 \rightarrow 0,41 \mathrm{~g} / \mathrm{cm}^{3}\right), 2\left(0,38 \rightarrow 0,43 \mathrm{~g} / \mathrm{cm}^{3}\right)$ e 3 $\left(0,37 \rightarrow 0,39 \mathrm{~g} / \mathrm{cm}^{3}\right)$ e decréscimo até a porção terminal do tronco nos 3 tratamentos, sem diferenças significativas, exceto a $12,0 \mathrm{~m}$ de altura no tratamento 2 , com os valores significativamente maiores em relação ao tratamento 1. Da mesma forma, a densidade básica média das árvores não apresentou diferenças significativas entre os três tratamentos (Tabela 2).

O modelo de variação longitudinal da densidade básica do lenho determinado para as árvores de eucalipto tem sido regularmente citado por diversos autores, como Barrichelo et al. (1983), Carpim et al. (1984), Lopes (2003) e Alzate (2004), sendo relacionado com as exigências mecânicas de sustentação do tronco e da copa das árvores de diferentes espécies de eucalipto.

A exemplo da densidade aparente do lenho na altura do DAP do tronco das árvores, a densidade básica média foi também menor do que a determinada por Souza et al. (1986) e Alzate (2004), em função da madeira adulta no tronco das árvores de eucalipto de idade mais avançadas (TOMAZELLO FILHO, 1985b, 1987).

Tabela 2. Variação da densidade básica do lenho no sentido longitudinal do tronco das árvores.

Table 2. Longitudinal wood basic density variation in eucalypt trees.

\begin{tabular}{|c|c|c|c|}
\hline \multirow{2}{*}{ Altura (m) } & \multicolumn{3}{|c|}{ Tratamento } \\
\hline & 1 & 2 & 3 \\
\hline 0,0 & 0,4144 a $(0,0236)$ & 0,4259 a $\quad(0,0166)$ & 0,4085 a $(0,0225)$ \\
\hline 1,3 & 0,3940 a $(0,0263)$ & 0,3919 a $\quad(0,0253)$ & 0,3791 a $(0,0264)$ \\
\hline 3,0 & 0,3928 a $(0,0238)$ & 0,3884 a $\quad(0,0245)$ & 0,3770 a $(0,0230)$ \\
\hline 6,0 & 0,3974 a $(0,0228)$ & 0,4043 a $(0,0381)$ & 0,3910 a $(0,0376)$ \\
\hline 9,0 & 0,4144 a $(0,0196)$ & 0,4324 a $\quad(0,0228)$ & 0,3940 a $\quad(0,0425)$ \\
\hline 12,0 & 0,3623 a $(0,0125)$ & $0,4262 \quad b(0,0195)$ & $0,3870 \mathrm{ab}(0,0139)$ \\
\hline Média & 0,3959 a $(0.0166)$ & 0,4115 a $\quad(0.0245)$ & $0,3894 \mathrm{a} \quad(0.0276)$ \\
\hline
\end{tabular}

Médias seguidas de desvio padrão. Numa mesma linha, médias seguidas de pelo menos uma letra igual não diferem

significativamente $(\mathrm{p}>0,05)$. Tratamento 1: testemunha; Tratamento 2: potássio; Tratamento 3: sódio. 


\section{Variação da estrutura anatômica do lenho: dimensões dos vasos e fibras}

A frequência dos vasos diminui no sentido radial do lenho das árvores de eucalipto dos 3 tratamentos, sendo de 31-34 vasos $/ \mathrm{mm}^{2}$ próximo à medula e de $10-13$ vasos $/ \mathrm{mm}^{2}$ próximo à casca. A diferença significativa ocorreu no terceiro $\mathrm{cm}$ do raio no tratamento $2\left(10 \mathrm{vasos} / \mathrm{mm}^{2}\right)$ em relação ao 1 e 3 (15 e 13 vasos $/ \mathrm{mm}^{2}$, respectivamente). O diâmetro tangencial dos vasos aumentou no sentido radial do lenho dos 3 tratamentos, de 46-51 a 89-104 $\mu \mathrm{m}$ da região próxima da medula à da casca, respectivamente, com diferenças significativas apenas no segundo e quarto $\mathrm{cm}$ do raio nos tratamentos $2 \mathrm{e}$ 3 (Tabela 3, Figura 5. Exemplo: tratamento 2).

Os valores e o modelo de variação radial determinado para o diâmetro e frequência dos vasos no lenho foram descritos para inúmeras espécies de eucalipto por Bamber e Humphreys (1969), Tomazello Filho (1985a,b, 1987, 2006), Foelkel et al. (1983), Leal et al. (2004) e Veenin et al. (2005), e relacionamse ao lenho funcional (alburno) e ao processo ascendente de fluxo da seiva bruta, e, da mesma forma, com as propriedades de utilização da madeira, afetando a penetração do licor e dos preservativos, a densidade e a qualidade superficial dos papéis de impressão, etc. (CHEN; EVANS, 2004).

A área do lenho ocupada pelos vasos apresenta uma tendência de aumento no sentido medulacasca nas árvores do tratamento 3, sendo que, nos demais, os valores são elevados próximo à medula $(11,8-12,6 \%)$, decrescendo até o primeiro-segundo $\mathrm{cm}(10,6 \%)$ e aumentando $(19 \%)$ em direção à casca (Tabela 3).

Apesar de significativos, o diâmetro do tronco (T1: 8,29 cm, T2: 10,66 cm e T3: 9,23 cm) e o volume de lenho (T1: $0,0325 \mathrm{~m}^{3}, \mathrm{~T} 2: 0,0644 \mathrm{~m}^{3}$ e T3: $0,0455 \mathrm{~m}^{3}$ ) das árvores de eucalipto nos 3 tratamentos, aos 24 meses, de acordo com as mensurações dendrométricas feitas por Sette $\operatorname{Jr}$ (2007), não apresentaram diferenças quanto aos elementos de vaso. Um idêntico modelo de variação para os elementos de vaso foi observado em árvores de eucalipto com 7 anos (TOMAZELLO FILHO, 2006), sendo, no entanto, observado um xilema funcional (alburno) de maior espessura nas árvores mais desenvolvidas e de maior área foliar.

Tabela 3. Dimensões dos vasos no sentido radial das amostras do lenho das árvores de eucalipto.

Table 3. Vessel dimensions in radial direction of the wood samples of eucalypt trees.

\begin{tabular}{|c|c|c|c|c|}
\hline \multirow{2}{*}{ Parâmetros } & \multirow{2}{*}{$\begin{array}{c}\text { Posição radial } \\
(\mathbf{c m})\end{array}$} & \multicolumn{3}{|c|}{ Tratamentos } \\
\hline & & 1 & 2 & 3 \\
\hline \multirow{6}{*}{$\begin{array}{l}\text { Frequência } \\
\left(\mathrm{n}^{\circ} / \mathrm{mm}^{2}\right)\end{array}$} & 0 & 33,74 a $(9,83)$ & 30,94 a $(10,42)$ & 34,40 a $(8,90)$ \\
\hline & 1 & 19,53 a $(1,97)$ & 20,43 a $(3,14)$ & 20,58 a $(2,37)$ \\
\hline & 2 & 15,76 a $(1,29)$ & 15,06 a $(1,73)$ & 14,01 a $(2,14)$ \\
\hline & 3 & 14,83 a $(2,38)$ & $10,33 \mathrm{~b}(1,41)$ & 12,61 a $(1,53)$ \\
\hline & 4 & 13,31 a $(1,26)$ & 11,27 a $(0,87)$ & 10,39 a $(1,23)$ \\
\hline & 5 & 13,66 a $(0,93)$ & 11,91 a $(1,95)$ & \\
\hline \multirow{6}{*}{$\begin{array}{l}\text { Diâmetro } \\
(\mu \mathrm{m})\end{array}$} & 0 & 51,46 a $(7,09)$ & 46,44 a $\quad(4,51)$ & 46,50 a $(5,67)$ \\
\hline & 1 & 64,21 a $(6,95)$ & 61,80 a $\quad(5,77)$ & 63,86 a $(4,63)$ \\
\hline & 2 & 75,28 a $(6,90)$ & 67,75 b $(5,87)$ & 80,73 a $(6,21)$ \\
\hline & 3 & 88,02 a $(6,95)$ & 83,87 a $\quad(9,67)$ & 90,42 a $(7,14)$ \\
\hline & 4 & 87,57 a $(2,26)$ & $102,31 \mathrm{ab}(8,89)$ & $107,47 \mathrm{~b}(4,74)$ \\
\hline & 5 & 89,00 a $(6,02)$ & 104,21 a $\quad(6,52)$ & \\
\hline \multirow{6}{*}{$\begin{array}{l}\text { Porcentagem da área } \\
(\%)\end{array}$} & 0 & 11,79 a $(1,14)$ & 12,64 a $(3,77)$ & 11,46 a $(1,08)$ \\
\hline & 1 & 10,61 a $(2,01)$ & 12,50 a $(2,08)$ & 12,09 a $(0,80)$ \\
\hline & 2 & 11,91 a $(1,51)$ & 10,59 a $(1,55)$ & 13,11 a $(1,20)$ \\
\hline & 3 & $13,98 \mathrm{ab}(2,83)$ & $11,16 \mathrm{~b}(0,74)$ & 15,24 a $(1,86)$ \\
\hline & 4 & 15,12 a $(1,23)$ & 17,23 a $(1,02)$ & 18,86 a $(1,77)$ \\
\hline & 5 & 18,88 a $(2,03)$ & 18,59 a $(1,29)$ & \\
\hline
\end{tabular}

Médias seguidas de desvio padrão. Numa mesma linha, médias seguidas de pelo menos uma letra igual não diferem significativamente $(\mathrm{p}>0,05)$. Tratamento 1: testemunha; Tratamento 2: potássio; Tratamento 3: sódio.

As referências bibliográficas sobre o efeito da fertilização mineral na dimensão e frequência dos elementos de vasos do lenho de eucalipto são escassas na literatura, restringindo a discussão dos resultados obtidos no presente trabalho. Bamber et al. (1982) reportaram a formação de vasos de menor 
diâmetro e de menor frequência no lenho de árvores de Eucalyptus grandis de 2,5 anos, induzidas a altas taxas de crescimento pela aplicação de fertilizantes. Por outro lado, Andrade et al. (1994) detectaram vasos de maior diâmetro no lenho de árvores de E. grandis de 6 anos, pela calagem do solo, e Tomazello Filho (2006) não observou significativas diferenças nas dimensões dos vasos em árvores de E. grandis $x$ urophylla fertilizadas e irrigadas.

A avaliação dos elementos de vaso e a formação do cerne e do alburno nas árvores de eucalipto, em idades mais avançadas, são fundamentais para se entender o mecanismo de fluxo ascendente de seiva bruta no xilema funcional do tronco das árvores de diferentes volumes de lenho e de área foliar, submetidas aos 3 tratamentos de fertilização.
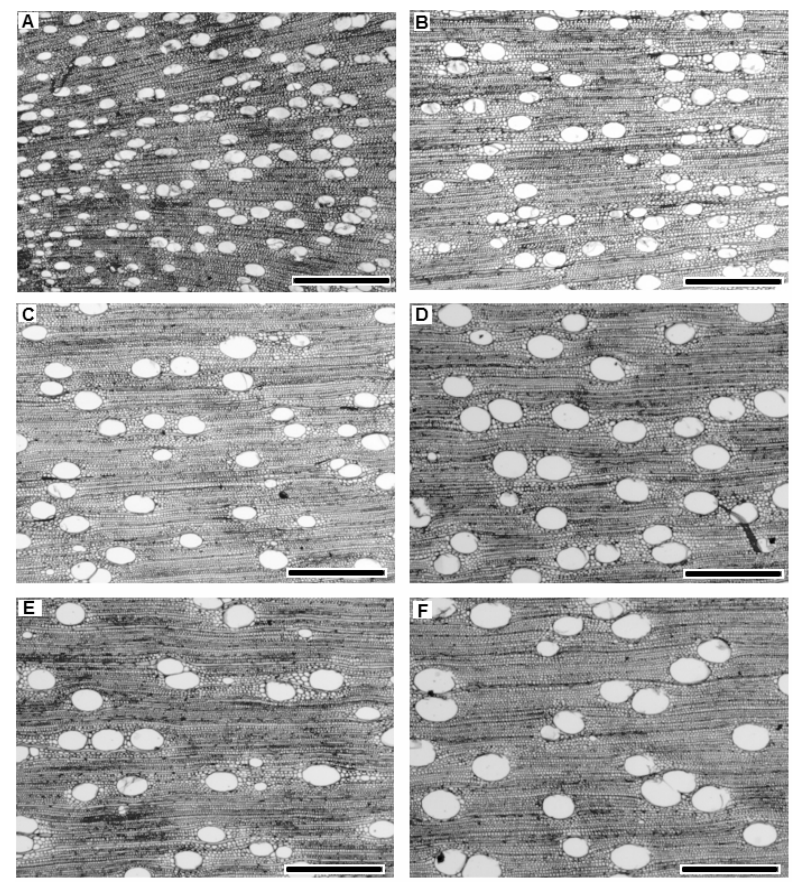

Figura 5. Variação do diâmetro tangencial e da frequência dos vasos no lenho das árvores de eucalipto em 6 posições radiais, da medula (A) até a casca (F). Aumento: $50 \mathrm{x}$. Barra $=200 \mu \mathrm{m}$.

Figure 5. Variation of the tangential diameter and frequency of the vessels in eucalypt wood, in 6 radial positions, from the pith (A) to the bark (F). $50 \mathrm{x}$. Bar $=200 \mu \mathrm{m}$.

No sentido medula-casca das amostras do lenho das árvores de eucalipto, as fibras de menor largura $(16,1-17,4 \mu \mathrm{m})$ estão próximas à medula, e as de maior largura $(17,9-19,3 \mu \mathrm{m})$ próximas à casca (Figura 6), sem apresentar diferenças significativas entre os tratamentos, exceto a 50\% do raio, com valor significativamente maior nos tratamentos 2 e 3 (Tabela 4). Esse modelo de variação radial da largura das fibras foi verificado por Dadswell (1958), Ferreira (1972), Thomas (1985), Tomazello Filho (1985b), Maeglin (1987), Oliveira (1997), Florsheim et al. (2000) e Rocha et al. (2002), sendo considerado, dessa forma, comum nas espécies de eucalipto.

O diâmetro do lume das fibras mostrou tendência de aumento no sentido radial das amostras do lenho dos eucaliptos (Figura 6), sendo significativamente menor a 50\% do raio no tratamento $1(9,7 \mu \mathrm{m})$ em relação aos tratamentos 2 e 3 (11,5 e 11,7 $\mu \mathrm{m}$, respectivamente) (Tabela 4). Esse comportamento é apontado como um dos vários modelos de variação (TOMAZELLO FILHO, 1985b; FLORSHEIM et al., 2000; ROCHA et al., 2002).

Com relação à espessura da parede das fibras não foi visualizado um modelo de variação (Figura 6), com diferenças significativas a $50 \%$ do raio nos tratamento 2 e $3(3,7$ e $3,4 \mu \mathrm{m})$ e a $100 \%$ nos tratamentos 1 e 2 (3,5 e 3,9 $\mu$ ) (Tabela 4). Diversos autores, como Ferreira (1972), Taylor (1973), Foelkel et al. (1983), Tomazello Filho (1985a,b, 1987) e Alzate (2004) indicam um aumento no valor da espessura da parede das fibras no sentido medula-casca, no lenho de árvores de eucalipto de maior idade. 
Tabela 4. Variação das dimensões das fibras no sentido radial das amostras do lenho das árvores de eucalipto.

Table 4. Variation of fiber dimensions in radial direction of the eucalypt wood.

\begin{tabular}{|c|c|c|c|c|}
\hline \multirow{2}{*}{ Parâmetros } & \multirow{2}{*}{$\begin{array}{c}\text { Posição radial } \\
(\%)\end{array}$} & \multicolumn{3}{|c|}{ Tratamentos } \\
\hline & & 1 & 2 & 3 \\
\hline \multirow{3}{*}{$\begin{array}{l}\text { Largura } \\
(\mu \mathrm{m})\end{array}$} & 0 & 16,13 a $(2,92)$ & 17,38 a $(3,60)$ & 16,63 a $\quad(3,23)$ \\
\hline & 50 & 16,73 a $\quad(2,67)$ & $18,95 \mathrm{~b}(3,06)$ & $18,51 \mathrm{~b}(3,23)$ \\
\hline & 100 & 17,81 a $\quad(3,13)$ & 19,09 a $(3,54)$ & 19,28 a $\quad(3,49)$ \\
\hline \multirow{3}{*}{$\begin{array}{l}\text { Diâmetro do lume } \\
(\mu \mathrm{m})\end{array}$} & 0 & $8,74 \mathrm{a} \quad(2,51)$ & 9,60 a $(3,05)$ & 9,61 a $\quad(2,49)$ \\
\hline & 50 & $9,72 \mathrm{a} \quad(2,21)$ & $11,47 \mathrm{~b}(2,45)$ & $11,74 \mathrm{~b}(2,87)$ \\
\hline & 100 & 10,91 a $\quad(2,61)$ & 11,25 a $(3,21)$ & $12,00 \mathrm{a} \quad(2,80)$ \\
\hline \multirow{3}{*}{$\begin{array}{l}\text { Espessura da parede } \\
(\mu \mathrm{m})\end{array}$} & 0 & 3,70 a $\quad(0,77)$ & 3,73 a $(0,98)$ & 3,51 a $\quad(0,64)$ \\
\hline & 50 & $3,50 \mathrm{ab}(0,66)$ & 3,74 a $(0,59)$ & $3,39 \mathrm{~b}(0,56)$ \\
\hline & 100 & 3,45 a $\quad(0,67)$ & $3,92 \mathrm{~b}(0,60)$ & $3,64 \mathrm{ab}(0,81)$ \\
\hline \multirow{3}{*}{$\begin{array}{l}\text { Comprimento } \\
(\mu \mathrm{m})\end{array}$} & 0 & 558,15 a $\quad(129,73)$ & 651,62 b $(273,35)$ & $610,46 \mathrm{ab}(120,34)$ \\
\hline & 50 & $728,44 \mathrm{ab}(140,78)$ & 805,39 a $(205,06)$ & $717,88 \mathrm{~b}(123,39)$ \\
\hline & 100 & 785,57 a $\quad(168,25)$ & 926,10 b $(171,31)$ & 932,68 b $(193,16)$ \\
\hline
\end{tabular}

Médias seguidas de desvio padrão. Numa mesma linha, médias seguidas de pelo menos uma letra igual não diferem significativamente $(\mathrm{p}>0,05)$. Tratamento 1: testemunha; Tratamento 2: potássio; Tratamento 3: sódio.
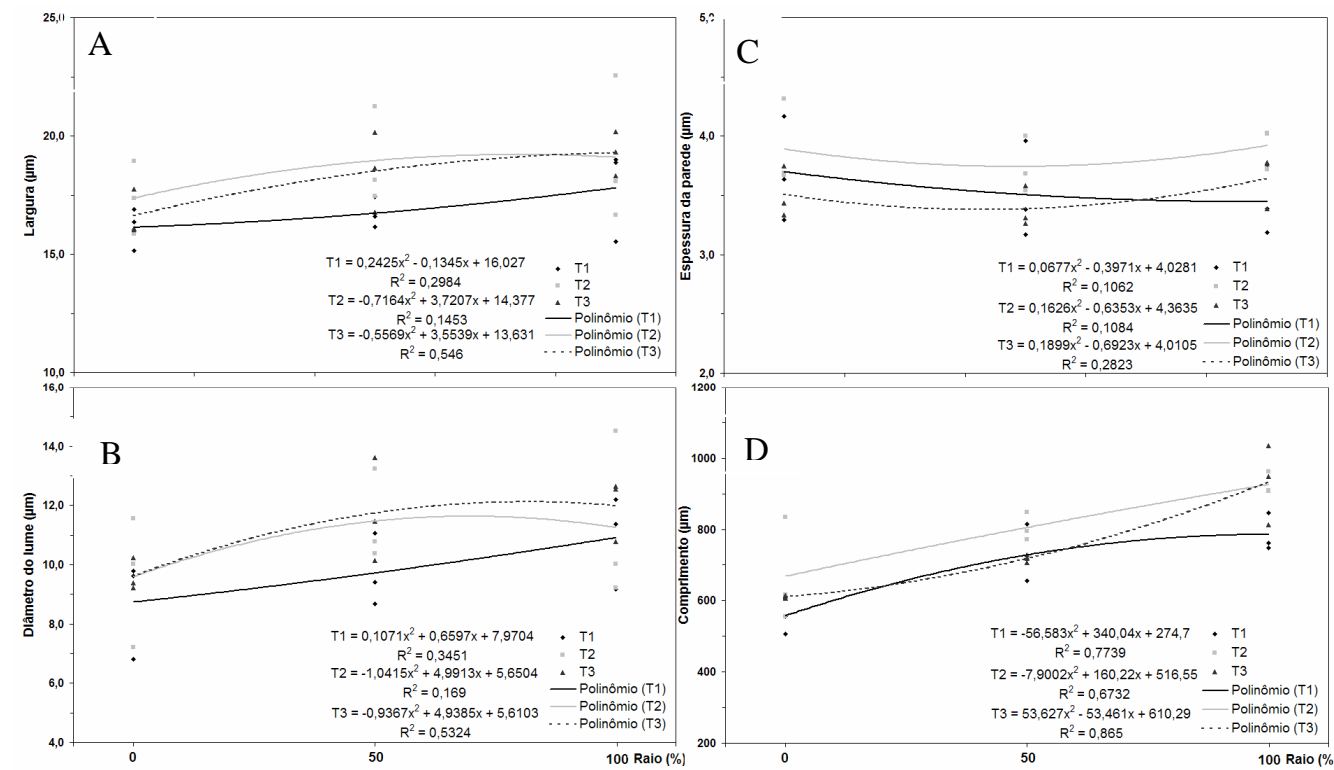

Figura 6. Variação das dimensões das fibras no sentido radial das amostras do lenho das árvores de eucalipto: largura (A), diâmetro do lume (B), espessura da parede (C) e comprimento (D). Tratamento 1: testemunha; Tratamento 2: potássio; Tratamento 3: sódio.

Figure 6. Variation of the fiber dimensions in the radial direction of the wood samples eucalypt tress: width (A), lume diameter (B), wall thickness (C) and length (D). T1: control; T2: potassium; T3: sodium.

O comprimento das fibras mostrou tendência de aumento no sentido radial, com diferenças nas regiões próximas à medula e à casca para os tratamentos $1 \mathrm{e} 2 \mathrm{e}$ posição intermediária nos tratamentos $2 \mathrm{e}$ 3 (Figura 6, Tabela 4). As referências da literatura, como Ferreira (1972 e 1979), Taylor (1973), Foelkel et al. (1983) e Tomazello Filho (1985a, b, 1987), indicam, de modo geral, que o comprimento das fibras aumenta no sentido medula-casca no lenho de árvores de eucalipto em idade avançada.

As fibras apresentaram maiores dimensões, de modo geral, no lenho das árvores de eucalipto adubadas com potássio e sódio, embora nem sempre significativos (Tabela 4), devendo esse comportamento estar relacionado com as maiores dimensões das árvores nesses tratamentos, conforme destacado por Sette Jr. (2007), conferindo maior sustentação ao tronco nas árvores mais desenvolvidas. 


\section{CONCLUSÕES}

Pode-se concluir que as árvores de eucalipto mostraram um perfil radial de densidade aparente do lenho característico de madeira juvenil e sem diferenças entre os três tratamentos. No sentido longitudinal, a densidade básica do lenho apresentou um modelo de variação caracterizado pelo decréscimo da base do tronco até 3,0 m; seguido de aumento até $9 \mathrm{~m}$ e decréscimo até o topo das árvores, comum a algumas espécies de eucalipto. Os elementos anatômicos do lenho, elementos de vaso e fibras mostraram modelos de variação no sentido medula-casca, descritos para algumas espécies de eucalipto. Para os elementos de vaso não foram observadas diferenças entre os tratamentos, no entanto para algumas dimensões das fibras detectaram-se diferenças no lenho das árvores de eucalipto, devendo isso estar relacionado com a sustentação do seu tronco e copa.

\section{REFERÊNCIAS}

ALZATE, S. B. A. Caracterização da madeira de árvores de clones de Eucalyptus grandis, E. saligna e E. grandis $\mathbf{x}$ urophylla. 133 p. Tese (Doutorado em Recursos Florestais - Tecnologia de Produtos Florestais) Escola Superior de Agricultura "Luiz de Queiroz”, Universidade de São Paulo, Piracicaba. 2004.

ANDRADE, A. M.; VITAL, B. R.; BARROS, N. F.; LUCIA, R. M. D.; CAMPOS, J. C. C.; VALENTE, O. F. Efeitos da fertilização mineral e da calagem do solo na produção e na qualidade da madeira de eucalipto. Revista Árvore, Viçosa, v. 18, n. 1, p. 69-78, jan./abr. 1994.

BAMBER, R. K.; HORNE, R.; GRAHAM-HIGGS, A. Effect of fast growth on the wood properties of Eucalyptus grandis. Australian Forest Research, Canberra, v. 12, n. 2, p. 163-167. Dec. 1982.

BAMBER, R. K.; FLOYD, A. G.; HUMPHREYS, F. R. Wood properties of flooded gum. Australian Forestry, Yarralumla, v. 33, n. 1, p. 3-12, 1969.

BARRICHELO, L. E. G.; BRITO, J. O.; MIGLIORINI, A. J. Estudo da variação longitudinal da densidade básica de Eucalytpus spp. Silvicultura, São Paulo, v. 8, n. 28, p. 726-31, jan./fev. 1983.

BEADLE, C.; BANHAM, P. W.; WORLEDGE, D.; RUSSEL, S. L.; HETERINGTON, S. J.; HONEYSETT, J. L.; WHITE, D. Effect of irrigation on growth and fibre quality of Eucalytpus globulus and Eucalyptus nitens. Appita Journal, Carlton, v. 54, n. 2, p. 144-147, 2001.

BENJAMIN, C. A. Estudo da estrutura anatômica e das propriedades físicas e mecânicas da madeira de corymbia (eucalyptus) citriodora e Eucalyptus grandis. 158 p. Tese (Doutorado em Agronomia - Energia na Agricultura) - Faculdade de Ciências Agronômicas - Universidade Estadual Paulista "Júlio Mesquita Filho", Botucatu, 2006.

BOUVET, J. M.; BOUILlET, J. P.; VIGNERON, P.; OGNOUABI, N. Genetic and environmental effects on growth and wood basic density with two Eucalyptus hybrids. In: CONGRESS ON CONNECTING BETWEEN SILVICULTURE AND WOOD QUALITY, 1999, Nancy. Proceedings... Nancy: IUFRO, 1999. p. 15-19.

BOX, G. E. P.; COX, D. R. An analysis of transformations. Journal of Royal Statistical Society, Series B, London, v. 26, p. 211-246. 1964.

CARPIM, M. A.; BARRICHELO, L. E. G. Influência da procedência e classe de diâmetro sobre as características da madeira de Eucalyptus grandis. In: CONGRESSO ANUAL DE CELULOSE E PAPEL, 17., 1984, São Paulo. Anais... São Paulo: ABCP, 1984. p. 23-34.

CHEN, F.; EVANS, R. A robust approach for vessel identification and quantification in eucalypt pulpwoods. In: APPITA ANNUAL CONFERENCE, 2004, Canberra. Proceedings ... Canberra: APPITA, 2004. p. 4.

DADSWELL, H. E. Wood structure variations occuring during tree growth and their influence on properties. Journal Institute of Wood Science, Madison, v. 1, p. 2-23, 1958. 
FERREIRA, M. Variação da densidade básica da madeira de povoamentos comerciais de Eucalyptus grandis nas idades de 11, 12, 13, 14 e 16 anos. IPEF, Piracicaba, n. 4, p. 65-89, jun.1972.

FERREIRA, C. A.; FREITAS, M.; FERREIRA, M. Densidade básica da madeira de plantações comerciais de eucaliptos na região de Mogi Guaçu. IPEF, Piracicaba, n. 18, p. 106-117, jun. 1979.

FLORSHEIM, S. M. B.; COUTO, H. T. Z.; SPEGIORIN, L.; ROCHA, F. T. Variação da estrutura anatômica da madeira de Eucalyptus saligna aos 7 anos. Revista do Instituto Florestal, São Paulo, v. 12, n. 2, p. 179-191, dez. 2000.

FOELKEL, C. E. B.; BUSNARDO, C. A.; DIAS, C.; SCHIMDT, C.; SILVA, R. M. R.; VESZ, J. B. Variabilidade radial da madeira de Eucalyptus saligna. Silvicultura, São Paulo, v. 8, n. 28, p. 782-791, 1983.

GAVA, J. L. Efeito de adubação potássica em plantios de E.grandis conduzidos em segunda rotação em solos com diferentes teores de potássio trocável. Série Técnica IPEF, Piracicaba, v. 11, n. 30, p. 89-94, abr. 1997.

IAWA COMMITTEE. IAWA list of microscopic features for hardwood identification. Wheeler, E. A., Baas, P; GASSON, P. E. (Ed.). IAWA Bulletin, Leiden, v. 10, n. 3, p. 219-332, 1989.

JACOB, W. S.; BALLONI, E. A. Efeitos da fertilização na qualidade da madeira. Boletim Informativo IPEF, Piracicaba, v. 6, n. 20, p. c1-13, nov. 1978.

LACLAU, J. P.; POGGIANI, F. RANGER, J.; GONÇALVES, J. L. M.; STAPE, J. L.; LIMA, W. P. de. Processos de transferência e balanço de água e de nutrientes em povoamentos de Eucalyptus que receberam aplicações de nitrogênio e de biossólido: reflexos sobre a sustentabilidade. 2007. 65 p. Relatório apresentado a Fundação de Amparo à Pesquisa do Estado de São Paulo - FAPESP.

LEAL, S.; PEREIRA, H.; GRABNER, M.; WIMMER, R. Clonal and site variation of vessels in 7 year old Eucalyptus globulus. IAWA Journal, Leiden, v. 24, n. 2, p. 185-195, 2004.

LOPES, M. C. Agrupamento de árvores matrizes de Eucalyptus grandis em função das variáveis dendrométricas e das características tecnológicas da madeira. 104 p. Dissertação (Mestrado em Engenharia Florestal - Tecnologia de Produtos Florestais) - Universidade Federal de Santa Maria, Santa Maria, 2003.

MAEGLIN, R. R. Juvenile wood, tension wood, and growth stresses effects on processing hardwoods. In: APPLYING THE LATEST RESEARCH HARDWOODS: PROCEEDINGS OF THE ANNUAL HARDWOOD SYMPOSIUM OF THE HARDWOOD RESEARCH COUNCIL, 15., 1987, Memphis. Proceedings... Memphis: Hardwood Research Council, 1987. p. 100-108.

MELlO, H. A. Aspectos do emprego de fertilizantes minerais no reflorestamento de solos de cerrado do estado de São Paulo com Eucalyptus saligna. 176 p. Tese (Mestrado em Silvicultura) Escola Superior de Agricultura “Luiz de Queiroz”, Universidade de São Paulo, Piracicaba, 1968.

MOTHE, F.; DUCHANOIS, G.; ZANNIER, B.; LEBAN, J. M. Microdensitometric analysis of wood samples; data computation method used at Inra-ERQB (Cerd program). Annals dês Foresters Sciences, Nanci, v. 55, n. 3, p. 301-313, 1998.

OLIVEIRA, J. T. S. Caracterização da madeira de eucalipto para a construção civil. 429 p. Tese (Doutorado em Engenharia Civil) - Escola Politécnica, Universidade de São Paulo, São Paulo, 1997.

PEREIRA, H.; ARAUJO, C. Raw-material quality of fast grow Eucalyptus globulus during the first year. IAWA Bulletin, Leiden, v. 11, n. 4, p. 421-427, 1990.

RAYMOND, C. A. Wood properties, silviculture and growth: a review of published literature for eucalypts. Cooperative Research Centre for Sustainable Production Forestry (Technical report, 6). Australia: CRC, 1998. 18 p.

ROCHA, F. T.; FLORSHEIM, S. M. B.; COUTO, H. T. Z.; SPEGIORIN, L. Variação da estrutura anatômica da madeira de Eucalyptus grandis aos sete anos de idade. In: ENCONTRO EM MADEIRA E EM ESTRUTURA DA MADEIRA, 7., 2002, Uberlândia. Anais... Uberlândia: 2002. p. 1-10. 
SAS INSTITUTE. SAS/STAT User's Guide: vertion 6.08. Carrey, 1997. v. 2.

SASS, J. E. Botanical microtechnique. $2^{\text {nd }}$ ed. Iowa: State College Press, 1951. 228 p.

SETTE JUNIOR, C. R. Efeito da aplicação do lodo de esgoto e de fertilização mineral no crescimento e propriedades da madeira de árvores de Eucalyptus grandis W. Hill ex. Maiden. 152 p. Dissertação (Mestrado em Recursos Florestais, com opção em Tecnologia de Produtos Florestais) Escola Superior de Agricultura “Luiz de Queiroz”, Universidade de São Paulo, Piracicaba, 2007.

SHIMOYAMA V. R. de S.; BARRICHELO, L. E. G. Importância da adubação na qualidade da madeira e celulose. In: SIMPÓSIO SOBRE ADUBAÇÃO E QUALIDADE DOS PRODUTOS AGRÍCOLAS, 1, 1989, Ilha Solteira. Anais... São Paulo: Icone, 1989. p. 1-15.

SILVA, J. C. Caracterização da madeira de Eucalyptus grandis de diferentes idades visando sua utilização na industria moveleira. 160 p. Tese (Doutorado em Engenharia Florestal) - Setor de Ciências Agrárias, Universidade Federal do Paraná, Curitiba, 2002.

SILVA, J. C.; OLIVEIRA, J. T. S.; TOMAZELLO FILHO, M.; KEINERT JR.; S.; MATOS, J. L. M. Influência da idade e da posição radial na massa específica da madeira de Eucalyptus grandis Hill ex. Maiden. Floresta, Curitiba, v. 34, n. 1, p. 13-22, jan./abr. 2004.

SILVEIRA, R. L. V. A.; MALAVOLTA, E. Produção e características químicas da madeira juvenil de progênies de Eucalyptus grandis em função das doses de potássio na solução nutritiva. Scientia Forestalis, Piracicaba, n. 63, p. 115-135, jun.2003.

SOUZA, V. R.; CARPIM, M. A.; BARRICHELO, L. E. G. Densidade básica entre procedências, classe de diâmetro e posições em árvores de Eucalyptus grandis e E. saligna. IPEF, Piracicaba, n. 33, p. 65-72, ago. 1986.

TAYLOR, F. W. Anatomical wood properties of South African Eucalyptus grandis. South African Forestry Journal, África do Sul, n. 84, p. 20-24, $1973 \mathrm{a}$.

THOMAS, R. J. The characteristics of juvenile wood. In: SYMPOSIUM ON UTILIZATION CHANGES ON WOOD RESEARCH OUTH US, 1985. Proceedings... Raleigh: North Carolina State University, 1985. p. 1-18.

TOMAZELLO FILHO, M. Estrutura anatômica da madeira de oito espécies de eucalipto cultivadas no Brasil. IPEF, Piracicaba, n. 29, p. 25-36, abr. 1985a.

TOMAZELLO FILHO, M. Variação radial da densidade básica e da estrutura anatômica da madeira do Eucalyptus saligna e Eucalyptus grandis. IPEF, Piracicaba, n. 29, p. 37-45, abr. 1985 b.

TOMAZELLO FILHO, M. Variação radial da densidade básica e da estrutura anatômica da madeira do Eucalyptus globulus, E. pellita e E. acmenioides. IPEF, Piracicaba, n. 36, p. 35-42, ago. 1987.

TOMAZELLO FILHO, M. Efeito da irrigação e fertilização nas propriedades do lenho de árvores de Eucalyptus grandis $\boldsymbol{x}$ urophylla. p. 135. Tese (Livre-Docência). Escola Superior de Agricultura "Luiz de Queiroz", Universidade de São Paulo, Piracicaba, 2006.

VEENIN, T.; FUJITA, M.; NOBUCHI, T.; SIRIPATANADILOK, S. Radial variation of anatomical characteristics and specific gravity in Eucalyptus camaldulensis clones. IAWA Journal, Leiden, v. 26, n. 3, p. 356-361, 2005.

VIGNERON, J.; GERARD, J.; BOUVET, J. M. Relationship between basic density and growth in a fertilization trial with clones of Eucalyptus hybrids in Congo. In: IUFRO CONFERENCE EUCALYPT PLANTATIONS: IMPROVING FIBRE YIELD AND QUALITY, 1995, Hobart. Proceedings ... Hobart: IUFRO, 1995. p. 68-71.

WASHUSEN, R.; BAKER, T.; MENZ, D.; MORROW, A. Effect of thinning and fertilizer on the cellulose crystallite width of Eucalyptus globulus. Wood Science and Technology, Berlin, v. 39, n. 7, p. 569-578, out. 2005. 